\title{
JUAN DE LUGO Y LA LIBERTAD EN ECONOMÍA. EL ANÁLISIS ECONÓMICO ESCOLÁSTICO EN TRANSICIÓN
}

\author{
FABIO MONSALVE SERRANO* \\ ÓSCAR DE-JUAN**
}

Resumen: Odd Langholm reconoce en Lugo ciertas ideas que lo presentan como un autor en transición entre el paradigma escolástico y el de los filósofos del derecho natural. El elemento central de esta transición es la despersonalización u objetivación de la economía caracterizada por la desaparición de la dimensión interpersonal en las relaciones económicas y de la necesidad como condicionante de la voluntad y de la validez de los contratos. Estas ideas se hacen patentes en Lugo en el tratamiento de la voluntad y en el del cumplimiento de los contratos. En este trabajo se revisa la tesis de Langholm concluyendo que en el primer caso la ruptura no es radical, pero sí en el segundo. En cualquier caso, las «nuevas ideas» en Lugo son un intento de armonizar la nueva realidad económica con la tradición escolástica, a la que pertenece.

Palabras clave: Historia del pensamiento económico, posición dominante, ética y justicia.

Abstract: Following Odd Langholm certain ideas of Lugo presents this scholar as an author in transition between scholastic and natural law philosophers paradigms. The key point is the depersonalization or the objectivization of the economy characterized by the disappearance of the interpersonal economic relations and the necessity as a condition of the will and of the covenants validity. These ideas appear on Lugo writings over the will and the keeping covenants. This paper revise Langholm thesis. The authors agreeing over the second item, not fully over the first one. Anyway, this «new ideas» are a Lugo attempt to fit the new economic reality with the scholastic legacy, where he belongs to.

Key words: History of Economic Thought, dominant position, ethics and justice.

Códigos JEL: B11, D42, Z10.

${ }^{*}$ ) Profesor Asociado del área de Economía Española e Internacional, Universidad de Castilla-La Mancha, Facultad de Ciencias Sociales de Cuenca.

(**) Catedrático de Teoría Económica, Universidad de Castilla-La Mancha, Facultad de Ciencias Económicas y Empresariales de Albacete. 
I. PODER, ELECCIÓN, NECESIDAD Y COACCIÓN EN ECONOMÍA

\begin{abstract}
Por mucho que Henri-Maximilien pretendiera sacudirse el polvo del mostrador familiar, no en balde se es hijo de un hombre que puede subir o bajar el precio de los productos, y que concede préstamos a los príncipes. La madre del héroe en ciernes le llenó la bolsa de vituallas y le dio a escondidas dinero para el viaje.
\end{abstract}

(Marguerite Yourcenar, Opus nigrum)

Henri-Maximilien, hijo de un mercader de Brujas, decide abandonar su casa natal abandonando al mismo tiempo su porvenir como hijo de mercader. La renuncia de Henri-Maximilien en términos económicos y de poder es particularmente importante y difícilmente podríamos buscarle una comparación en la actualidad; y esto no por el hecho de que su padre prestase dinero a los príncipes, sino por el hecho de que podía subir y bajar el precio de los productos. En un entorno de agentes económicos precio-aceptantes, la capacidad de fijar los precios queda reservada a pocas empresas y esto, además, como una circunstancia transitoria pues ese poder tiende a desaparecer en el largo plazo.

Concede Marguerite Yourcenar, pues, al mercader la capacidad de alterar el precio de los productos. Para poder explicar ese poder hemos de tener en cuenta dos elementos que marcan una diferencia sustancial con la sabiduría económica convencional actual.

En primer lugar, la realización de las transacciones económicas en un marco de relaciones interpersonales. Comprador y vendedor concurrían individualmente y su voluntad y decisión eran determinantes para concluir la transacción. 
The modern mechanistic conception of the market as a suprapersonal force setting the terms to which an individual exchanger must submit was foreign to the medieval masters. Their frame of reference was a moral universe that obliged any buyer or seller to act for the common good and agree to terms of exchange accordingly, regardless of the advantage granted him by the forces of the market. This means that the common estimate of the just price could not refer indiscriminately to whatever price might be obtainable under existent market conditions. (Langholm 1998: 85)

En segundo lugar, la necesidad de vender o de comprar o de tomar un préstamo, en un entorno de ausencia de un mercado sistematizado, podía llevar a los agentes económicos a tomar decisiones que de otro modo no hubieran tomado. Así pues, la voluntad queda condicionada por la necesidad.

Son estos dos elementos los que conceden veracidad a la frase de que el mercader podía subir o bajar el precio de los productos. Así pues, el mercader de Brujas, en su doble condición de comerciante y banquero adinerado, no tenía necesidad de vender a precios que él no decidiera. No se encontraría en la situación de urgencia de vender para poder subsistir.

Ahora bien, la mayoría de los agentes económicos no gozaban de esa privilegiada situación. La necesidad podía condicionar su voluntad en las transacciones económicas y desde la postura más débil verse sometidos a las condiciones que quisiera imponer la más fuerte. En este caso, la compulsión derivada de la necesidad impediría la voluntariedad en la transacción.

Como es sabido, en el esquema escolático se exigía el respeto a la justicia conmutativa en las relaciones contractuales, de tal manera que ninguna de las partes quedara en peores condiciones que la otra tras haber realizado la operación. La justicia conmutativa se basaba, pues, en una «razón aritmética» según la cual las partes contratantes han de intercambiar bienes equivalentes. 
El peligro para el respeto de la justicia que suponía la necesidad del comprador -o vendedor- era evidente. La parte más débil obligada por su necesidad no tendría más remedio que consentir. Su libertad quedaría condicionada. Así pues, necesidad, compulsión, voluntad y libertad son elementos presentes en las transacciones económicas que motivaron la reflexión de los doctores escolásticos por su incidencia sobre la moral y sobre la justicia.

Esta problemática, a la que tanto esfuerzo y tratados dedicaron los doctores escolásticos, constituye el objeto central del excelente libro de Odd Langholm, The legacy of scholasticism in economic thought. Antecedents of choice and power. ${ }^{1}$ En este estudio Langholm reflexiona sobre el concepto de libertad en Economía, la capacidad de elegir y la limitación que el poder y la necesidad pueden ejercer sobre la misma. Para ello emprende un recorrido por toda la tradición económica escolástica -desde Santo Tomás de Aquino hasta Juan de Lugo- buscando las conexiones etimológicas, jurídicas y teológicas a través de las cuáles se va construyendo la doctrina de los doctores en torno a la libertad de elección en economía. ${ }^{2}$

Langholm sostiene dos tesis fundamentales en este estudio. La primera es considerar que la aportación principal y más duradera de la corriente escolástica a la Economía ha sido su reflexión sobre el concepto de libertad económica.

${ }^{1}$ Entre los historiadores y analistas del pensamiento económico de la escolástica, Odd Langholm se ha situado en un lugar preeminente. Con sus estudios sobre el precio justo, el dinero y la usura ha tratado de reconstruir el camino que conecta a Aristóteles con los doctores escolásticos medievales, así como las influencias que sobre éstos ejercieron las fuentes romanistas, patrísticas y canonísticas.

2 En este sentido, conviene recordar que el paradigma escolástico no puede considerarse como un bloque monolítico. Las discrepancias entre autores eran lugar común. Sucesivamente se introducía nuevos matices en las disputas que reorientaban el problema en un intento permanente de adecuación a la cambiante realidad económica. Advierte, pues, Langholm sobre «the common error of regarding the scholastic tradition as too uniform, overlooking the breakdown of the medieval paradigm at the hands of the late scholastics and their gradual assumption of some of the premises of the new economics». (Langholm 1998: 176) 
In scholastic economic thought, freedom in that sense brought to bear on two of the elements of all market economies, namely, the phenomenon of need in the face of scarcity and the arrangement of need satisfaction by means of exchange. The question of freedom then became the question of the state of the will of a person who consents to certain terms of exchange because of need. Conversely, it became the question of compulsion by need or, in a personalized sense, of compulsion (or coercion) by one party to the exchange taking advantage of the other party's need. (Langholm 1998: vii)

La segunda es que en la transición entre el paradigma escolástico y el de los filósofos del derecho natural -en el seno del cual Smith sistematizaría sus ideas- se produciría el inicio de la objetivación o despersonalización de la economía. ${ }^{3}$ Esta despersonalización se caracterizaría, en primer lugar, porque desaparece la dimensión interpersonal en las relaciones económicas y con ello la posibilidad de exigir una responsabilidad moral en las mismas. En segundo lugar, deja de ser relevante la voluntariedad con que los agentes acuden al mercado, $y$, por tanto, la necesidad como condicionante de la transacción. Se modifica así el concepto de libertad económica. A partir de entonces la justicia desaparece como virtud moral. Si en la tradición aristotélica la justicia de los contratos se sustentaba en los términos, con Hobbes ésta pasa a sustentarse en el cumplimiento. Se trata pues de un cambio crucial, en la medida en que la cultura occidental se ha construido en torno al cumplimiento de los contratos.

Junto con las dos ideas anteriores y en conexión con ellas, Langholm muestra como dentro de la tradición escolástica es posible detectar algunas ideas que conectan a estos autores con los posteriores filósofos del derecho natural. De entre estos

${ }^{3}$ El término objetivación hay que atribuírselo a Gordon (1975: 270). Langholm prefiere hablar de despersonalización. 


\section{autores Langholm reserva un lugar especial a Lugo pues con- sidera que en su análisis sobre la usura}

Lugo makes two important observations... Both are devastating to the scholastic doctrine and point forward to another era, already dawning elsewhere in Europe. (1998: 75)

Es nuestro propósito en este artículo tratar de analizar más detenidamente la postura de Juan de Lugo, el Doctor Hispalense, y con ello revisar la importante tesis de Langholm al respecto del final del periodo de la escolástica y su enlace con los filósofos del derecho natural. ${ }^{4}$ Esta revisión nos permitirá, además, profundizar en lo señalado hasta ahora sobre los conceptos de necesidad, compulsión, voluntariedad y libertad. Con-

${ }^{4}$ Juan de Lugo es comúnmente considerado como el último gran representante del renacer escolástico español, al menos en lo que a pensamiento económico se refiere (Grice-Hutchison 1982: 135; Roover 1957: 119). Dentro de las distintas tradiciones escolásticas (véase nota 2), Lugo se inserta en aquella que surge en España en el siglo XVI a través de Francisco de Vitoria y sus seguidores. En esta corriente se inserta Lugo; en ella se forma; de ella toma los temas de estudio, el método de análisis, la forma de entender y abordar la realidad, la preocupación moral en la investigación... Es su escuela, su ambiente intelectual. Pertenece, pues, al grupo que denominaremos «doctores escolásticos españoles de los siglos XVI y XVII». Schumpeter se refirió a ellos como «Escolásticos tardíos» (Schumpeter 1994: 134), y Grice-Hutchison, con la expresión más exitosa de «Escuela de Salamanca» (Grice-Hutchison 1982). El término «Escuela de Salmanca» ha suscitado alguna controversia (Grice-Hutchison 1993: 25-27).

Juan de Lugo, nació en Madrid en 1583. Su infancia la pasó en Sevilla, quedando fuertemente vinculado a esta ciudad, de ahí que firme sus obras como «Ionnes de Lugo Hispalensis». En el año 1603 ingresó en la Compañía de Jesús. Su labor dentro de la orden fue eminentemente docente, impartiendo clases en Medina del Campo, Monforte de Lemos, León, Salamanca y Valladolid. En el año 1621 es reclamado por el General de la Compañía para ocupar la Cátedra de Teología escolástica del colegio que la Compañía tenía en Roma. En 1643 es nombrado Cardenal por el papa Urbano VIII. Murió en Roma en 1660.

En relación con su obra, a nuestro objeto interesa reseñar el tratado De Iustitia et Iure, y más concretamente las disputas XXV -sobre la usura- y XXVI -sobre la compra-venta-. La primera edición apareció en Lyon en el año 1642. Le siguieron las de 1646, 1652 y 1670, publicadas también en Lyon. Las siguientes ediciones aparecen, en el marco de sus obras completas, en los años 1718 y 1751 -ediciones venecianas- y 1868 y 1891 -ediciones parisinas de Vivés-. 
ceptos relevantes para una aproximación no convencional a la realidad de las transacciones económicas y para reconstruir el camino de la despersonalización de la economía.

II.

\section{EL PAPEL DE LA VOLUNTAD EN EL ANÁLISIS ECONÓMICO ESCOLÁSTICO}

Al reflexionar sobre la virtud en su Moral a Nicómaco reconoce Aristóteles que ésta sólo puede predicarse de los actos voluntarios de ahí que sea

un estudio imprescindible cuando se quiere dar razón de la virtud determinar lo que debe entenderse por acto voluntario e involuntario (1997: 122)

Por acto involuntario entiende Aristóteles aquellos que se hacen por fuerza mayor o por ignorancia. Ahora bien, dentro de este grupo también podemos encontrarnos con actos que realizamos bien debido al temor de males mayores o bien por un motivo noble.

Por ejemplo: un tirano, dueño de vuestros padres y vuestros hijos, os impone una cosa vergonzosa; podéis salvar esas personas que os son queridas, si os sometéis; y perderlas, si rehusáis someteros; $y$, en un caso semejante, se puede preguntar si el acto es voluntario o involuntario. Algo análogo sucede al marino que en una tempestad arroja al mar las mercancías. En los casos ordinarios nadie que tenga buen sentido arroja al agua los bienes que posee, pero no hay hombre sensato que no esté dispuesto a hacerlo si es una condición precisa para salvarse él o salvar a los demás. Las acciones de este género son, puede decirse, acciones mixtas; sin embargo, se aproximan más a las acciones voluntarias. Son el resultado de una preferencia en el momento mismo en que se hacen, y el objeto definitivo del acto está en relación con las circunstancias... En los actos que 
acabamos de citar, se obra aún libremente, porque el principio que para estos actos pone en movimiento los miembros de nuestro cuerpo que los ejecutan está en nosotros; y siempre que está en nosotros sólo de nosotros depende hacer o no las cosas. $(1997: 124)^{5}$

Como se desprende de la cita, Aristóteles resuelve la cuestión de la voluntariedad de las acciones mixtas aduciendo que éstas son principalmente voluntarias pues la acción final no es sino una elección del agente dadas las circunstancias; y puesto que existe elección, existe voluntariedad.

Por otra parte, la tradición legal europea establecía el consentimiento como condición necesaria para la validez de los contratos. Si algún contrato era resultado de la fuerza o del miedo la voluntad del contratante quedaba anulada y no podía ser sancionado por la ley. Lo cual no implica que la compulsión fuera incompatible con el consentimiento. ${ }^{6}$

La duda surge, pues, en relación con la voluntad en caso de las acciones mixtas; es decir, de aquellas en las que existe cierta compulsión que obliga a tomar una decisión que en otras circunstancias no se hubiera tomado. Para clarificar la cuestión, los escolásticos distinguieron entre voluntad absoluta (simpliciter) y voluntad condicionada (secundum quid). En el primer caso no hay lugar a dudas. En el segundo, la tesis escolástica sancionó la de Aristóteles y fue taxativa a la hora de considerar que la compulsión no invalida la voluntariedad: ${ }^{7}$ el agente escoge

5 Este texto de Aristóteles, y concretamente el ejemplo del capitán, será profusamente citado y utilizado en toda la tradición escolástica.

${ }^{6}$ De hecho, en el Digesto pueden encontrarse dos posturas contradictorias sintetizadas en las siguientes afirmaciones de Paul y Ulpiano, respectivamente: «...although I should not have willed if I were free; still, being forced, I have willed»; «Nothing is a contrary to consent... as force and fear». Langholm afirma que, considerado en su conjunto, acabó dominando la primera. (1998: 34-37)

7 Esta severidad, si bien se ampara en las consideraciones de Aristóteles y de la tradición legal europea, no es ajena a la cuestión del pecado dentro de la moral cristiana. La libertad para pecar y para combatir el pecado es una premisa funda- 
voluntariamente una opción de entre las posibles dadas las circunstancias. Así pues, la voluntad forzada no deja de ser voluntad. Otra cuestión sería la mejor o peor disposición con la que actúe.

Esta severidad doctrinal admitió una excepción: el de la coacción o compulsión económica, entendida como

Economic compulsion is compulsion by a person's own need, utilized by another person to his advantage. (Langholm 1998: 6)

De hecho, Langholm considera que la piedra angular que sustenta y da consistencia lógica al edificio de análisis económico escolástico es la lucha contra la explotación de la necesidad económica individual. Es este leit motiv lo que sustantiva dicho análisis y marca la principal diferencia de la escolástica con otros paradigmas.

Una vez precisado el papel de la voluntad y la necesidad dentro de la doctrina escolática, retomamos el propósito del artículo sobre el papel de Lugo en el periodo final de la escolástica.

III.

\section{JUAN DE LUGO Y LA DESPERSONALIZACIÓN DE LA ECONOMÍA}

\section{Necesidad, voluntad y usura}

El préstamo de dinero y la usura fue la primera de las actividades económicas que centró la atención de los doctores escolásticos y sobre la que aplicaron sus ideas sobre la compulsión, la voluntad y la necesidad.

mental de la doctrina católica. Ahora bien, si la compulsión se considerara como un factor atenuante, se abrirían las puertas al pecado involuntario pero forzado por las circunstancias. Cosa que no podían admitir los teólogos escolásticos. 
La postura que prevaleció entre los autores de los siglos XIII y XIV fue la de que aunque el prestatario pague un interés por encima del principal -usura- éste no lo hace libremente sino movido de la necesidad, ya sea en sentido estricto -necesario para la existencia- o en sentido más amplio -necesario para existir de forma adecuada-. Para Sto. Tomás:

One who accepts a loan always suffers necessity either in the first or in the second of these two senses. If this is interpreted literally, it means that usury is always paid involuntarily, regardless of the purpose for which the money was intended, and that all usury is theft. (Citado en Citado en Langholm 1998: 64).

La usura es pues una forma de robo, pues el usurero toma algo que no le pertenece contra la voluntad de su legítimo propietario. El argumento de la compulsión se convirtió en una razón más en contra del cobro de usuras ${ }^{8}$ contra la devolución de algo por encima del capital prestado en el contrato de mutuo. ${ }^{9}$ Señala Langholm (1998: 65-66) que dicho argumento fue dejando de utilizarse durante los siglos XIV y XV y las discusiones sobre la usura se reorientaron hacia las circunstancias y los

${ }^{8}$ Los otros argumentos principales contra la usura serían: a) La esterilidad del dinero; b) el riesgo en la transferencia de propiedad; c) el consumo de los bienes con el uso; d) los beneficios sobre la industria del prestatario; y, e) la a-causalidad del tiempo. Cfr. Langholm (1984) y Monsalve (2002)

${ }_{9}$ Conviene recordar que la definición de usura sólo podía predicarse del contrato de mutuo: «Definiremos la usura como el lucro que proviene del mutuo de modo inmediato y como algo debido. Por ejemplo, si se exigieren ciento diez a cambio de cien que se entregaron en mutuo, a los cien que se entregan se llama capital o 'suerte' (sors), y a los otros diez se llama usura, interés, o ganancia sobre la 'suerte' o capital. Por consiguiente, no será usura si el lucro proviniera, no del mutuo, sino de una compra-venta, aunque fuera injusta; tampoco será usura si la cantidad no se diera como algo debido, sino por benevolencia, gratitud o amistad; se tratará sólo de mutuo formal, o virtual, cual se encuentra en la compra-venta en la que el bien se vende más caro por razón de haber diferido el pago del precio, o más barato por haberlo anticipado; pues en ambos casos interviene un mutuo virtual por razón del precio cuyo pago se retrasa o anticipa originando un lucro por diferencia con el precio justo.» (Lugo 25: 6). 
títulos extrínsecos (damnum emergenes, lucrum cessans) que legitimaban el cobro de un interés. Así mientras que la construcción doctrinal sobre la injusticia de la usura permanecía intacta las circunstancias atenuantes la sancionaban.

Veamos lo que escribe Lugo en relación con la voluntad y la usura.

...la usura, como se ha dicho en muchas ocasiones, es un pecado contra la justicia, para el que se requiere esencialmente que el prójimo sea gravado contra su voluntad o sufra algún daño. Así pues, cuando no existe daño ni se grava al prójimo no puede tratarse de pacto injusto ni, por tanto usurario. (Lugo 25: 55)

Ahora bien la voluntad podría verse condicionada por la necesidad.

...para juzgar si el pacto es o no usurario no basta con mirar sólo al daño que del pacto se pueda seguir para el mutuatario, sino también a la carga o daño que deberá padecer por aceptar la obligación pactada; pues aunque la ejecución del mismo no implique de hecho ningún daño, sin embargo, la obligación que se acepta, en cuanto contradistinta de la ejecución del pacto, es tal que el mutuatario podría someterse a ella involuntariamente, es decir, coaccionado por la necesidad que tiene del mutuo. Pues como quiera que toda la injusticia de la usura nazca de la involuntariedad con que se acepta, siempre que exista involuntariedad habrá usura. Sin embargo, cuando esa obligación se estime en nada, o se considere como inexistente por el que la acepta, cesará el fundamento para que exista usura e injusticia en la obligación contraída; pues se acepta de modo absoluto y plenamente voluntario, es decir, sin mezcla de involuntariedad. (Lugo 25: 55).

El problema se plantea al considerar si el prestatario paga el interés libremente o movido por la necesidad. Si lo hiciera libremente y teniendo en cuenta el principio aristotélico de que nadie sufre injusticia voluntariamente, parece claro que en este caso 
no habría injusticia en el pago de ese algo más por encima del capital. Es decir, si el prestatario pagara la usura voluntariamente entonces no existiría robo. Así pues, la voluntariedad se convierte en la clave del problema.

Frente a la firmeza de Sto. Tomás de considerar el pago siempre como involuntario, Lugo reconoce que respecto a la obligación de pago «el mutuatario podría someterse a ella involuntariamente»; el condicional nos indica que también podría pagar algo por encima del capital de forma voluntaria, como muestra de liberalidad y gratitud, en cuyo caso no se considera un préstamo usurario, pues lo que paga no es usura sino libre gratitud (Lugo 25: IV). Así pues la voluntad puede ser la de pagar y el pagar no necesariamente implica involuntariedad.

De esta forma Lugo rompe la conexión entre necesidad e involuntariedad. El prestatario puede querer un préstamo sin sufrir la necesidad de la que hablaba Sto. Tomás. Alguien que acepte un préstamo no siempre sufre necesidad. Este nuevo matiz que recoge el Doctor Hispalense frente a la tradición escolástica de los siglos XIII y XIV ha de relacionarse con los dos siguiente hechos:

- La variación de la realidad económica entre el estado cuasi-estacionario de los siglos XIII y XIV y el estado más económicamente dinámico de los siglos XVI y XVII.

- La variación en la concepción de los usos del dinero. Lugo ya no percibe el dinero como una mera mercancía que sirve para el intercambio de bienes. Su idea del dinero es más compleja. El dinero puede ser usado como medio de cambio y consumido o puede ser utilizado para la negociación y convertirse en capital. ${ }^{10}$

10 Lugo (28: 54) reconoce que en los mercaderes el «principal instrumento de negociación es el dinero». Al discutir sobre el lucro cesante, utiliza con frecuencia las expresiones invertir, negociar y beneficiarse con el dinero (Lugo 28: VI.1). 
Así pues el préstamo podría no deberse a la necesidad sino al deseo de negociar. El condicional se manifiesta como importante pues es el elemento que permite a Lugo mantener la construcción escolástica contra el abuso de poder y conectarla con la nueva realidad económica necesitada de mayor flexibilidad financiera.

Profundizando algo más en la cuestión podríamos preguntarnos qué pasa con la propiedad del dinero que se paga en concepto de usura. Si se admitiera que el dominio pasa al usurero la cuestión de la voluntad se torna irrelevante y, de hecho, se sancionaría el cobro de la usura. Al contrario, si se sostiene que tal dominio no pasa al usurero. Recordemos que según Langholm, al resolver esta cuestión,

Lugo makes two important observations... Both are devastating to the scholastic doctrine and point forward to another era, already dawning elsewhere in Europe. (1998: 75)

Antes de abordar la cuestión de la transferencia de dominio del pago de la usura conviene precisar que la cuestión que se plantea no es sobre la legitimidad del cobro de la usura -cuestión que queda fuera de duda- sino qué ocurre con el dominio de lo que se paga en concepto de usura, entendida como la devolución de algo más sobre el principal sin título que lo justifique (lucrum cessans, damnum emergens, riesgo, gratitud...). Es decir, una vez producido físicamente el pago, ¿de quién es el domino de las monedas?, ¿del usurero que las recibe ilegítimamente?, ¿del prestatario que las entrega de forma involuntaria?

Hablando sobre las prácticas de los banqueros, describe cómo los depósitos fructifican para el banquero: «No es menor, sino antes mayor el beneficio que se hace al banquero depositándole el dinero, con el que entretanto puede negociar y lucrarse. Más aún, los banqueros estiman en tanto el beneficio, que a veces añaden una remuneración al depositante» (Lugo 28: 63). 
Hubo autores, principalmente canonistas, que defendieron la transferencia de propiedad. En la tradición escolástica, la postura fue más bien la contraria. De entre los varios argumentos que unos y otros expusieron interesa detenernos en aquel que hace referencia a la voluntad.

Para los autores que defendían la transferencia de dominio, existe voluntad de pagar aunque esta sea condicionada (secundum quid) y no absoluta (simpliciter), pues es preferible la usura a un daño superior, en consecuencia sí existe transferencia de propiedad. ${ }^{11}$ Así pues, el pago de la usura habría que encuadrarlo dentro de las acciones mixtas de las que hablaba Aristóteles. Acciones consideradas como voluntarias, de lo cual se deduce la transferencia de dominio. Molina nos resume la postura de estos autores.

Porque el dominio de los bienes se adquiere por la entrega de los mismos, y allí se da esta razón: «porque nada hay tan natural a la equidad natural como el que el dueño tenga voluntad determinada de transferir una cosa a otro»; y como el prestatario entrega al prestamista el lucro usurero por propia voluntad, se sigue que el prestamista adquiere así el dominio de ese lucro o beneficio. Y no es obstáculo que esa entrega sea una acción involuntaria mixta, pues como dice Aristóteles es simplemente (simpliciter) voluntaria y sólo condicionalmente (secundum quid) involuntaria; de igual manera que la acción de quien forzado por la tempestad arroja su mercancía al mar sea considera simplemente voluntaria. (Molina 1989: 199)

Para otros autores, no es posible hablar de transferencia de dominio pues no existe título justo que lo legitime. Entre ellos Molina, quien responde al anterior razonamiento apoyándose en la idea de necesidad como compulsión.

11 Sobre estos autores véase Langholm (1998: 71-73). 
Aunque sea involuntaria condicionalmente (secundum quid), si se obligó a actuar así injustamente, por ejemplo, coaccionando con miedo a la donación de algo o al pago de un precio que no se justifica, tal involuntariedad no es suficiente para transferir el dominio, aunque al mismo tiempo se acción simplemente (simpliciter) voluntaria.

Aunque Molina considera que el usurero no adquiere el dominio de la usura con carácter general, sí lo puede hacer ocasionalmente (per accidens).

Ocasionalmente el usurero se puede convertir a veces en dueño del dinero que ganó mediante la usura. Así, por ejemplo, si ese dinero se mezclase con otro del usurero. (Molina 1989: 204)

Esta apreciación deriva de una concepción meramente física del dominio de las monedas. Obviamente si éstas se mezclan con las que lícitamente recibiera o tuviera el usurero, no podría distinguirse las unas de las otras y adquiriría el dominio de todas, aunque el prestatario retiene el derecho a reclamarlo y a que se le prefiera a otro acreedor.

Para Lugo (25: 206) «el usurero adquiere el dominio de las usuras, pero sólo un dominio débil y con obligación de restituir». Es decir, al pagar la usura existe un simple cambio de mano del dinero, pero la propiedad y el derecho a reclamarlo permanecen en el prestatario.

Sobre esta tesis del Doctor Hispalense Langholm afirma:

According to Lugo, there can be no question but that the ownership of money paid as usury passes to the usurer. Whether the coins in question are mingled with other coins or not is irrelevant. Lugo makes two important observations... Both are devastating to the scholastic doctrine and point forward to another era, already dawning elsewhere in Europe. (Langholm 1998: 74-5). 
Veamos detenidamente cuáles son esas dos observaciones.

La primera de estas observaciones se relaciona con la cuestión de la voluntad.

Aunque la voluntad esté mezclada con algo de involuntariedad, debe considerarse simplemente libre, no impidiendo que la transferencia sea eficaz el que en cierto aspecto exista involuntariedad. Porque, realmente, la involuntariedad con la que el mutuatario paga las usuras no es mayor que la involuntariedad con que paga el interés que se paga del daño emergente, y por eso no niega que el dominio del interés se transfiera eficazmente. (Lugo 25: 206)

A partir de esta cita de Lugo Langholm concluye que «this observation robs the Aristotelian model of most of its meaning as an instrument of economic analysis» (1998: 75). La involuntariedad que pudiera existir no invalida la transferencia de propiedad. Si el prestamista acudió movido por la necesidad o no es indiferente pues esto sólo afectaría al grado de involuntariedad o voluntariedad con que se somete al pago de la usura y esto, en definitiva, a partir de Lugo, es irrelevante.

Conviene, sin embargo, matizar esta apreciación de Langholm. Inmediatamente después del texto anterior, Lugo continúa.

Por tanto, si el dueño quiere transferir eficaz y absolutamente el dominio de lo que entrega, y no sólo cumplir con la acción externa de lo que entrega, no vemos razón por la que su voluntad no deba considerarse eficaz si no existe ley positiva que le impida a él transferirla, ni al usurero recibirla. (Lugo 25: 206).

Así pues Langholm parece fijarse solamente en la primera parte del texto en la que se habla sobre la hipotética involuntariedad del prestatario al pagar bien las usuras, bien el interés por el daño emergente. Si en el segundo caso es lícito, ¿por qué no en el primero? No obstante, Lugo continúa y pone el 
acento en que el dueño «quiere transferir eficaz y absolutamente el dominio de lo que entrega». Es en este caso en el que no hay duda sobre la transferencia de propiedad.

Además, Lugo es concluyente al inicio de toda esta discusión sobre su postura al respecto

También parece cierto que el dominio no se transfiere al usurero si el mutuatario las pagó sin intención de transferir su dominio, y lo hizo sólo para no faltar a su palabra. Por lo tanto, muchas veces habrá que reconocer que, de hecho, no se adquiere el dominio por el usurero, puesto que el mutuatario no tiene intención de hacerlo por ser consciente de que no debe pagarlas, y se limitó a entregarlas sólo por cumplir la palabra dada. (Lugo 25: 204).

El texto deja clara la idea de que la transferencia de propiedad depende de la intención con que el mutuatario entregue las usuras. En ciertas ocasiones si existirá y otras «muchas veces habrá que reconocer que, de hecho, no se adquiere el dominio por el usurero...»

Así pues, no parece que Lugo se desvincule plenamente del planteamiento de la compulsión y la voluntad como Langholm parece sostener. No obstante, aunque en la construcción doctrinal Lugo se mantenga fiel a la tradición de pensamiento en la que se inserta, la incorporación del matiz que desvincula la transferencia de propiedad de la voluntad no deja de tener su importancia y realmente supone incorporar un elemento extraño al análisis que cuestiona la congruencia del mismo. Si todo el modelo se sustenta en la cuestión de la voluntad, y la libertad económica que otorga la ausencia de necesidad, para evitar situaciones de abuso de poder, matizar dicha cuestión le resta parte de su consistencia. Lugo, educado en una tradición centenaria, está excesivamente atado a la misma como para desvincularse de forma radical. Percibió los retos que planteaba la nueva realidad y trató de responder a la misma mediante 
refinamientos en la argumentación y no mediante un cambio en el método y el enfoque. Éste sería el gran error, no sólo de Lugo, sino de los escolásticos en general y que privaría al análisis económico de buena parte de sus conclusiones.

Mais, l'impressionnant appareil dialectique ne peut plus dissimuler le fait que les scolastiques avaient épuisé les ressources de leur méthode qui, au lieu de plus de raffinement, avait besoin d'une transformation complète et d'une revisión de fond en comble. Malheureusement, les scolastiques n'en virent pas la nécessité et se refusèrent à évoluer avec le temps. Assaillis de tous côtés -par les Cartésiens, les Jansénistes, les Philosophes, les Encyclopédistes et les Physiocrates- ils se cramponnèrent malgré tout à leurs méthodes désuètes, et leurs doctrines économiques subirent le même sort que leur philosophie en tombant dans le discrédit le plus complet. (Roover 1971: 36-7).

La segunda de las observaciones de la que nos hablaba Langholm se relaciona con la cuestión del cumplimiento de los contratos.

El texto relevante en esta ocasión es el siguiente:

... como quiera que el mutuatario sepa muy bien que las usuras no las debe en justicia, y que el usurero no tiene derecho a ellas, no puede pretender pagar una verdadera deuda ya que no existe. Sólo podrá pretender saldar la deuda de la fidelidad humana a la palabra dada. Prometió transferir el dominio, aunque no de forma gratuita sino por el beneficio recibido con el mutuo, y aunque esto no sea merecedor de un precio, el mutuatario quiere dar por ello más de lo que en realidad vale sólo porque así lo prometió. (Lugo 25: 207).

A partir de esta cita Langholm concluye:

Lugo's treatise De iustitia et iure appeared in 1642. The very same year saw the publication of Hobbes's De cive. In this work, a novel idea of natural law finds expressions. Decisively 
breaking with Aristotle, one of its main tenets is than men fulfil their promises, even when proceeding from fear, for justice pertains to the keeping of covenants, not to their terms. (1998: 76)

En este caso coincidimos plenamente con la interpretación de Langholm al respecto de la importancia que el mantenimiento de los contratos - porque así lo prometió- adquiere sobre los términos de los mismos-aunque esto no sea merecedor de un precio-.

A raíz de todo lo anterior podemos concluir,

En primer lugar, que el cobro de algo más por encima del capital sin título que lo legitime es injusto y constituye el elemento central de la visión de Lugo sobre la cuestión de los préstamos y la usura. Ahora bien, quien solicita el préstamo puede necesitarlo para subsistir o bien desearlo por otros motivos menos imperativos como el de negociar. En una $u$ otras circunstancias el grado de coacción es distinto y distinta la voluntariedad con la que se somete a dicho préstamo y a las cargas usurarias. Lugo, como el resto de doctores escolásticos españoles de los siglos XVI y XVII, fue más proclive a pensar en términos de deseabilidad que de necesidad.

En segundo lugar, que la transferencia de propiedad de las usuras dependerá de la voluntad absoluta (simpliciter) con que las pague el prestatario:

- Si las paga voluntariamente bien por liberalidad y gratitud, bien por fidelidad a la palabra dada, no existe lugar a dudas sobre la transferencia de propiedad

- Si las paga sin intención de hacerlo, es claro que no se adquiere el dominio por el usurero o de hacerlo sólo se adquiere un dominio débil y con obligación de restituir.

La voluntad sigue siendo esencial en su planteamiento, como lo era con anterioridad. Sí hemos de reconocer, no obstante, que Lugo ve a un prestatario más dispuesto a pagar algo por encima 
del capital. La generalización y diversificación de las prácticas mercantiles hace que los límites entre la coacción y un razonable interés se difuminen, lo cual no implica que la intención no siga siendo determinante.

En tercer lugar, convenimos con Langholm que en Lugo se perfila la transición entre respeto a la justicia conmutativa -característico de la tradición aristotélica- y al cumplimiento de los contratos -característico del derecho natural-como pilar de las transacciones económicas. Esta transición obviamente no hay que entenderla como una ruptura brusca. Para Lugo la justicia conmutativa sigue siendo un elemento esencial como se desprende de su tratado sobre la compra-venta. No obstante, sí es posible apreciar la incorporación de ciertos matices de los que el texto citado es un buen ejemplo.

\section{Necesidad, voluntad y compra venta}

El tratamiento escolástico de la compra-venta gira nuevamente en torno al concepto de libertad económica con sus componentes de necesidad, voluntad y poder. La defensa que los doctores hicieron de la fijación de precios por la estimación común de mercado hay que enmarcarla dentro de ese esquema. ${ }^{12}$

In the medieval context, it makes more sense to interpret the market estimate of the just price, understood in the sense explained previously, as a means to combat the exploitation of individual economic need. (Langholm 1998: 88)

El análisis escolástico sobre el precio justo trata pues de evitar la explotación de la necesidad por parte del más poderoso.

12 En este sentido existe una amplia discusión sobre si el precio justo es el mismo concepto que el precio. Entre los que asimilan ambos conceptos tenemos a Grice-Hutchison, Chafuen y Roover . Entre los defensores de la diferenciación se sitúan Gómez Camacho y Langholm. 
Este principio rector hubo de confrontarse con tres principios del derecho romano que, en su formulación literal, lo negaban.

1. una cosa vale tanto cuanto puedes obtener por ella; $;^{13}$

2. quien voluntariamente consiente en algo no se le injuria; ${ }^{14} \mathrm{y}$,

3. cada uno es el moderador y árbitro de sus propias cosas. ${ }^{15}$

La asunción de estos principios strictu sensu concedería total libertad contractual a los agentes económicos. La parte más poderosa podría aprovecharse de la necesidad del débil. La capacidad de elección quedaba limitada. Por otra parte, estos principios tornaban irrelevante la cuestión de la voluntariedad. Todo esto llevó a un reinterpretación de estos principios por parte de los doctores escolásticos para adecuarlos a sus planteamientos morales y económicos (Langholm 1982).

La adecuación del primer principio se hizo de dos modos: reescribiéndolo y modificándolo. En el primer principio -el más devastador con respecto a la cuestión del precio justo- el verbo poder se asocia al significado de deber; por lo tanto, el poder adquiere una connotación moral y no factual. Por otra parte, ya en la tradición salmantina, Domingo de Soto modificó dicho principio para aclarar el concepto: una cosa vale tanto cuanto puedes obtener por ella en ausencia de fuerza, fraude y engaño, razones que anularían la voluntad del comprador. La modificación de Soto, enlazaría con el segundo principio y la cuestión de la voluntad. Se señala que el consentimiento debe ir acompañado de la ausencia de fuerza, fraude o engaño, en cuyo caso no existiría voluntariedad. Además, la necesidad puede obligar a una parte a

\footnotetext{
13 «Res tantum valet quantum vendi potest». Digesto 9, 2, 33.

14 «Volenti ac consentienti non fit injuria». Digesto 39, 3, 1.

15 «In re sua uniquisque est moderator et arbiter». Código de Justiniano 4, 35,21
} 
consentir en un contrato injusto, lo cual ha de interpretarse también como involuntariedad, pues estaríamos hablando de un consentimiento condicionado por la fuerza de las circunstancias. Finalmente, el tercer principio se reinterpreta afirmando que sólo existe total libertad sobre el precio de los bienes si éstos no son necesarios, es decir, son bienes de lujo. Vemos como los tres preceptos se refinan para incorporales las significaciones que dotan de sentido a la construcción escolástica, tal y como venimos señalando. Así, la dimensión moral se incorpora en el primero, la cuestión de la voluntad en el segundo y la de la necesidad en el tercero.

Lo presentado hasta el momento sería la tradición dominante en la corriente escolástica. Sin embargo, en el siglo XVI Cayetano introduce un matiz que sería ampliamente seguido y que supone un punto de inflexión en el tratamiento de la necesidad y la compulsión económica que afecta a la voluntariedad. Cayetano distingue entre el modo de compra-venta y la causa de la misma (Langholm 1998: 112ss). Para Cayetano la justicia ha de fijarse atendiendo al primero y no a la segunda (modus than causa). De esta forma, el individuo ha de someterse a la estimación común del precio sin que sus circunstancias particulares influyan en el mismo. Esta distinción hace que se desvanezca la relevancia de la necesidad y la compulsión en el contrato de compra-venta. El énfasis se pone en que cuando se concurre al mercado se hace libremente y supone un mutuo acuerdo entre ambas partes. Si el precio es justo, el acuerdo es justo y la transacción es justa.

La justicia del contrato de compra y venta depende fundamentalmente de la justicia del precio, pues la igualdad entre los contratantes, objeto de la justicia, no se guarda cuando el precio es superior o inferior al justo. Hay que explicar, pues, cuál sea el precio justo que el comprador debe pagar y el vendedor debe exigir. (Lugo 26: 38) 
El acento pues hay que ponerlo en garantizar la justicia del precio y evitar situaciones de abuso de poder tales como la especulación, la colusión o el monopolio. Ésta es la mejor garantía para evitar la explotación económica de la necesidad individual.

En relación con la voluntad y el modus than causa planteado por Cayetano encontramos en Lugo una postura similar.

También crece este valor vulgar o natural por la escasez de lo que se vende, por la abundancia que hay de dinero, y decrece por los motivos contrarios. No basta, sin embargo, para aumentar el precio la mayor necesidad que el comprador pueda tener del bien, o la oportunidad que tendrá de obtener de él una mayor ganancia. Por tanto, no puede venderse el pan más caro a un hambriento por el simple hecho de que tenga hambre el comprador, ni un haz de hierbas comunes se puede vender más caro porque el comprador conozca el secreto para obtener de ellas un medicamento valioso y apreciado, pues estas circunstancias no varían la común estima de los bienes... Finalmente, el precio vulgar o natural varía dependiendo del modo en que se compran o venden los bienes. Se suele vender más caro cuando se venden al por menor que cuando se venden al por mayor; porque el primer modo de venta acarrea mayores gastos y trabajos al vendedor, por lo que se sube el precio de venta con razón. También se venden más baratos los objetos que el vendedor ofrece espontáneamente y de forma insistente que los que se compran en el almacén del mercader, pues las mercancías no buscadas o demandadas bajan su precio en una tercera parte... (Lugo 26: 43-44)

Por tanto, si la causa -la necesidad-con la que se acude a la realización de la compra-venta no es relevante, también deja de serlo la compulsión y la voluntariedad. La defensa de la parte más débil hay que buscarla en la justicia en el precio y no en la especial atención a unas circunstancias particulares.

Así pues, la prevalencia del modo sobre la causa introduce un nuevo elemento de despersonalización de la realidad 
económica. En esta cuestión Lugo se manifiesta como continuador de la tradición escolástica desde Cayetano.

IV.

\section{CONCLUSIONES}

A lo largo de la tradición escolástica la compulsión no se consideró como un atenuante de la voluntad, en la medida en que, en consonancia con la tesis de Aristóteles sobre las acciones mixtas, el agente siempre actuaba eligiendo entre diversas alternativas, dadas unas circunstancias. Otra cuestión era que esas circunstancias le fueran adversas. Así pues, la voluntad condicionada (secundum quid) no dejaba de ser voluntad. Esta severidad, sin embargo, no era aplicable al campo de la economía, donde la necesidad como compulsión sí se consideró como un elemento atenuante de la voluntad y por tanto susceptible de invalidar los contratos. La interpretación más plausible de esta divergencia de tratamiento radica en la defensa de la parte más débil de la transacción económica. La construcción doctrinal escolástica tuvo la finalidad de combatir la explotación de la necesidad económica del agente individual. En un entorno histórico en que las relaciones económicas eran relaciones interpersonales con un alto componente individual la amenaza del abuso por parte del más poderoso era una realidad presente.

Lugo se mantuvo fiel a esta tradición, sin embargo, el diferente entorno mercantil y financiero del siglo XVII le obligó introducir matizaciones que armonizaran el corpus escolástico con la nueva realidad.

En relación con la usura tres de ellas son especialmente relevantes. En primer lugar, la consideración de que el préstamo puede solicitarse por necesidad o por otros muchos motivos, de ahí que devolver algo por encima del principal no haya de 
ser siempre involuntario. La concepción del dinero como capital no es ajena a este cambio. En segundo lugar, la consideración de que cierto grado de involuntariedad no invalida la eficacia de los contratos. La relevancia que Langholm concede a esta idea conviene matizarla con otros textos en los que Lugo si defiende claramente la importancia de la voluntad. En tercer lugar, la consideración de que la justicia de los contratos va a depender no tanto de los términos del mismo cuanto de su cumplimiento.

Estas tres ideas cuestionan el pilar central del edifico escolástico en relación con la necesidad, la voluntad y la coacción y cuestionar este pilar se traduce en una pérdida de consistencia lógica de todo el análisis económico escolástico.

En cualquier caso, y pese a las anteriores «ideas revolucionarias» la figura de Lugo no puede entenderse como la de un revolucionario del pensamiento económico escolástico. Lugo se mantuvo fiel a la tradición en la que se educó y su metodología, presupuestos, principios y principales conclusiones fueron los de aquella. Sí percibió algunos de los nuevos retos pero su fidelidad le llevó a tratar de armonizarlos mediante refinamientos dialécticos y no mediante un cambio radical.

\section{REFERENCIAS BIBLIOGRÁFICAS}

Aristóteles (1997), Moral, a Nicómaco. Madrid: Espasa-Calpe. ANDRADE, A. de (1666), Varones ilustres en santidad, letras y zelo de almas de la Compañía de Jesús. Vol. V, Madrid.

Dempsey, B. W. (1948), Interest and Usury. London: Dennis Dobson Ltd.

Fuentes Quintana, E. (dir.) (1999), Economía y economistas españoles. Vol. II. Barcelona: Galaxia Gutenberg-Círculo de Lectores. 
Gómez Camacho, F. (1998), Economía y filosofía moral: La formación del pensamiento económico europeo en la Escolástica española. Madrid: Síntesis.

- (2004), Espacio y tiempo en la Escuela de Salamanca: el tratado de J. de Lugo, S.J. sobre La Composición del Contínuo. Salamanca: Universidad de Salamanca, Fundación VargasZúñiga y Pérez-Lucas.

Gómez Camacho, F. y Robledo, R. (eds.) (1998), El pensamiento económico en la Escuela de Salamanca. Salamanca: Ediciones Universidad de Salamanca.

Gordon, B. (1975), Economic analysis before Adam Smith: Hesiod to Lessius. London: Macmillan.

Grice-Hutchison, M. (1982), El pensamiento económico en España: 1177-1740. Barcelona: Crítica. [Versión castellana de Early economic thought in spain 1177-1740. Londres: George Allen \& Unwin. 1978.]

- (1993), Economic thought in spain: Selected essays of Marjorie Grice-Hutchison. Cambridge: Edward Elgar Publishing. Langholm, O. (1979), Price and value in the Aristotelian tradition. Bergen: Universitetsforlaget.

- (1982), «Economic freedom in scholastic thought». History of Political Economy, Vol 14, n ${ }^{\circ} 2$, pp. 260-283.

- (1983), Wealth and money in the aristotelian tradition: A study in scholastic economic sources, Bergen: Universitetsforlaget.

- (1984), The Aristotelian analisys of usury. Oslo: Universitetsforlaget.

- (1998), The legacy of scholasticism in economic thought: Antecedents of choice and power. Cambridge: Cambridge University Press.

Lugo, J. de (1868), De iustitia et iure, en VIVÉS, L., (ed.), Disputationes scholasticae et morales, TT. VI y VII, Parisiis.

Molina, L. de (1981), La teoría del justo preci., Madrid: Editora Nacional. [Edición moderna de F. Gómez Camacho.] 
- (1989), Tratado sobre los préstamos y la usura. Madrid: ICI, Instituto de Estudios Fiscales. [Edición moderna de F. Gómez Camacho.]

Monsalve, F. (2002), El pensamiento económico de Juan de Lugo. Un estudio de sus teorías del precio justo, del dinero y del interés. Tesis Doctoral. Universidad de Castilla-La Mancha. Albacete.

NARedo, J.M. (1996), La economía en evolución: Historia y perspectivas de las categorías básicas del pensamiento económico. Madrid: Siglo XXI.

Noonan, J.T. (1957), The scholastic analysis of usury. Cambridge: Harvard University Press.

Pribram, K. (1983), A history of economic reasoning. Baltimore: Johns Hopkins University Press.

ROOver, R. de (1971), Le pensée économique des scholastiques: doctrines et Méthodes. Montreal-París: Institutes d’Études Mediévales-J.Vrin.

Rothbard, M. (1999), Historia del Pensamiento Económico. Madrid: Unión Editorial.

Schumpeter, J.A. (1994), Historia del análisis económico. Ariel: Barcelona. [Versión castellana de History of economic análisis. Oxford: Oxford University Pres, Inc., 1954.]

Sмith, A. (1999), Investigación sobre la naturaleza y causa de la riqueza de las naciones. Barcelona: Folio. [Versión castellana de The Wealth of Nations, 1776.]

Vigo, A. del (1997), Cambistas, mercaderes y banqueros en el siglo de oro español. Madrid: B.A.C. 